\title{
Susceptibility of Bacterial Endophthalmitis Isolates to Vancomycin, Ceftazidime, and Amikacin
}

\author{
Kuan-Jen Chen ( $\nabla$ cgr999@gmail.com ) \\ Chang Gung Memorial Hospital \\ Ming-Hui Sun \\ Chang Gung Memorial Hospital \\ Chiun-Ho Hou \\ Chang Gung Memorial Hospital \\ Hung-Chi Chen \\ Chang Gung Memorial Hospital

\section{Yen-Po Chen} \\ Chang Gung Memorial Hospital

\section{Nan-Kai Wang \\ Columbia University}

\section{Laura Liu \\ Chang Gung Memorial Hospital} \\ Wei-Chi Wu \\ Chang Gung Memorial Hospital \\ Hung-Da Chou \\ Chang Gung Memorial Hospital

\section{Eugene Yu-Chuan Kang} \\ Chang Gung Memorial Hospital

\section{Chi-Chun Lai} \\ Chang Gung Memorial Hospital
}

\section{Research Article}

Keywords: Bacterial endophthalmitis, Susceptibility, Vancomycin, Ceftazidime, Amikacin, rare intraocular infection

Posted Date: July 8th, 2021

DOI: https://doi.org/10.21203/rs.3.rs-677943/v1

License: (a) (1) This work is licensed under a Creative Commons Attribution 4.0 International License. Read Full License

Version of Record: A version of this preprint was published at Scientific Reports on August 5th, 2021. See the published version at https://doi.org/10.1038/s41598-021-95458-w. 


\section{Abstract}

Bacterial endophthalmitis is a rare intraocular infection, and prompt administration of intravitreal antibiotics is crucial for preventing severe vision loss. The retrospective study is to investigate the in vitro susceptibility to the antibiotics vancomycin, amikacin, and ceftazidime of bacterial endophthalmitis isolates in specimens at a tertiary referral center from January 1996 to April 2019 in Taiwan. Overall, 450 (49.9\%) isolates were gram positive, 447 (49.6\%) were gram negative, and $4(0.4 \%)$ were gram variable. In gram-positive isolates, coagulase-negative staphylococci were the most commonly cultured bacteria (158, $35.1 \%)$, followed by streptococci $(100,22.2 \%)$, enterococci $(75,16.7 \%)$, and Staphylococcus aureus $(70,15.6 \%)$. In gramnegative isolates, they were Klebsiella pneumoniae (166, 37.1\%) and Pseudomonas aeruginosa (131, 29.3\%). All grampositive organisms were susceptible to vancomycin, with the exception of one Enterococcus faecium isolate (1/450, $0.2 \%)$. Of the gram-negative isolates, $96.9 \%$ and $93.7 \%$ were susceptible to ceftazidime and amikacin, respectively. Nine isolates (9/447, 2.0\%) were multidrug-resistant gram-negative bacteria, comprising Klebsiella pneumoniae (4/164, 2.4\%), Acinetobacter baumannii $(2 / 3,67 \%)$, and Stenotrophomonas maltophilia (3/18, $17 \%)$. In conclusion, in vitro susceptibility testing revealed that vancomycin remains the suitable antibiotic treatment for gram-positive endophthalmitis. Ceftazidime and amikacin provide approximately the same degree of gram-negative coverage. Multidrug-resistant bacterial endophthalmitis was uncommon.

\section{Background}

Bacterial endophthalmitis is a rare intraocular infection that can occur following ocular surgery or trauma, as well as through the hematogenous spread of microorganisms from endogenous infection. Prompt administration of intravitreal antibiotics with or without pars plana vitrectomy is crucial for preventing severe vision loss. Both the bacterial spectrum and antibiotic susceptibility patterns in bacterial endophthalmitis isolates must be considered in the context of treatment. Vancomycin is a first-line drug for managing gram-positive bacterial endophthalmitis, whereas ceftazidime or amikacin are typically used for gram-negative coverage. Amid growing concern over the emergence of multidrug-resistant (MDR) bacteria, selecting antibiotics for infection treatment has become a critical issue. Common MDR organisms include (1) vancomycin-resistant enterococci; (2) methicillin-resistant Staphylococcus aureus; (3) extended-spectrum $\beta$-lactamase (ESBL)-producing gramnegative bacteria; (4) carbapenemase-producing Klebsiella pneumoniae, and (5) MDR gram-negative bacteria, such as Klebsiella pneumoniae, Enterobacter spp., Escherichia coli, Acinetobacter baumannii, Pseudomonas aeruginosa, and Stenotrophomonas maltophilia. This group of gram-positive and gram-negative bacteria has been named the ESKAPE group (Enterococcus faecium, S. aureus, K. pneumoniae, A. baumannii, P. aeruginosa, and Enterobacter spp.) ${ }^{1}$. Studies have been conducted on MDR bacterial endophthalmitis ${ }^{2-8}$. Although most isolates causing ocular infection are not caused by MDR bacteria, antibiotic susceptibility testing may reveal trends in antimicrobial susceptibility and resistance.

This study investigated the in vitro susceptibility of gram-positive and gram-negative bacterial endophthalmitis isolates collected over 23 years to vancomycin, ceftazidime, and amikacin. MDR pathogens were also examined.

\section{Methods}

The protocol of this retrospective, noncomparative laboratory case series was approved by the Institutional Review Board of Chang Gung Memorial Hospital in Taiwan, and the requirement for written informed consent from the patients from whom the specimens were collected was waived. All clinical procedures were conducted according to the principles of the Declaration of Helsinki. The microbiological data of bacterial cultures isolated from culture-confirmed bacterial endophthalmitis at the Department of Microbiology of the participating hospital between January 1996 and April 2019 were reviewed. To protect the patients' privacy, the data were deidentified. Data on clinical presentations and visual outcomes were not part of the laboratory records.

All microbiology investigations were performed at Microbiology Department, Chang Gung Memorial Hospital, Taoyuan, Taiwan. Bacterial culture isolates were identified by conventional microbiological methods (January 1996 to December 2013) 
and matrix-assisted laser desorption/ionization time of flight mass spectrometry (MALDI-TOF-MS) (January 2014 to April 2019). Conventional microbiological methods included Gram-staining and biochemical tests. In MALDI-TOF-MS, automatic measurement of the spectrum and comparative analysis with reference spectra of bacteria were performed using an Ultraflextreme mass spectrometer and MALDI-Biotyper 3.0 software (Bruker Daltonics). The reliability of identification in the MALDI Biotyper system was expressed in points. A log(score) $\geq 2.0$ indicated identification to the species level. The isolates were tested for susceptibility to various antibiotics using the Kirby Bauer Disc diffusion method on Mueller Hinton blood agar. The Clinical and Laboratory Standards Institute (Wayne, PA) standards were used for interpretation and quality control for each corresponding year ${ }^{9}$. In vitro susceptibility of cultured bacterial organisms, the tested antibiotics mostly included either vancomycin for gram-positive bacteria or ceftazidime and amikacin for gram-negative bacteria. All data from the antibiotic susceptibility testing were further reviewed in the gram-positive bacterial isolates resistant to vancomycin and in the gramnegative bacteria resistant to either ceftazidime or amikacin. To be considered MDR, organisms must be resistant to antimicrobial drugs of three or more classes. Although methicillin-resistant $S$. aureus (MRSA) was susceptible to vancomycin, MRSA was not defined as an MDR organism in this study.

\section{Results}

A total of 901 bacterial endophthalmitis isolates were cultured over the 23-year study period. An overview of the isolates is provided in Table 1. 
Table 1

Bacteria Isolated from Patients Diagnosed with Endophthalmitis. Note: CoNS = coagulasenegative Staphylococcus; NF-GNB = non-fermentative-gram-negative bacilli

\begin{tabular}{|c|c|c|c|}
\hline Gram-positive & Genus or Species & 450 & $49.9 \%$ \\
\hline \multirow[t]{8}{*}{ Cocci } & Staphylococcus spp. & & \\
\hline & CoNS & 158 & $17.5 \%$ \\
\hline & S. aureus & 70 & $7.8 \%$ \\
\hline & Streptococcus spp. & 100 & $11.1 \%$ \\
\hline & Enterococcus spp. & 75 & $8.3 \%$ \\
\hline & Aerococcus spp. & 1 & $0.1 \%$ \\
\hline & Micrococcus spp. & 3 & $0.3 \%$ \\
\hline & Rothia mucilaginosa & 1 & $0.1 \%$ \\
\hline \multirow[t]{5}{*}{ Bacilli } & Corynebacterium spp. & 9 & $1.0 \%$ \\
\hline & Cutibacterium acnes & 10 & $1.1 \%$ \\
\hline & Paenibacillus spp. & 1 & $0.1 \%$ \\
\hline & Bacillus spp. & 18 & $2.0 \%$ \\
\hline & Clostridium spp. & 4 & $0.4 \%$ \\
\hline Gram-negative & & 447 & $49.6 \%$ \\
\hline Cocci & Neisseria spp. & 4 & $0.4 \%$ \\
\hline Coccobacilli & Hemophilus spp. & 11 & $1.2 \%$ \\
\hline \multicolumn{4}{|l|}{ Bacilli } \\
\hline \multirow[t]{8}{*}{ Enterobacteriaceae } & Klebsiella spp. & 166 & $18.4 \%$ \\
\hline & Citrobacterspp. & 8 & $0.9 \%$ \\
\hline & Enterobacter spp. & 16 & $1.8 \%$ \\
\hline & Morganella spp. & 2 & $0.2 \%$ \\
\hline & Proteus spp. & 12 & $1.3 \%$ \\
\hline & Salmonella spp. & 2 & $0.2 \%$ \\
\hline & Serratia spp. & 12 & $1.3 \%$ \\
\hline & Escherichia spp. & 13 & $1.4 \%$ \\
\hline Non-Enterobacteriaceae- & Aeromonas spp. & 3 & $0.3 \%$ \\
\hline fermentative & Vibrio spp. & 1 & $0.1 \%$ \\
\hline Non-Enterobacteriaceae- & Acinetobacterspp. & 5 & $0.6 \%$ \\
\hline \multirow[t]{3}{*}{ nonfermentative } & Burkholderia spp. & 4 & $0.4 \%$ \\
\hline & Flavobacterium spp. & 3 & $0.3 \%$ \\
\hline & Moraxella spp. & 4 & $0.4 \%$ \\
\hline
\end{tabular}




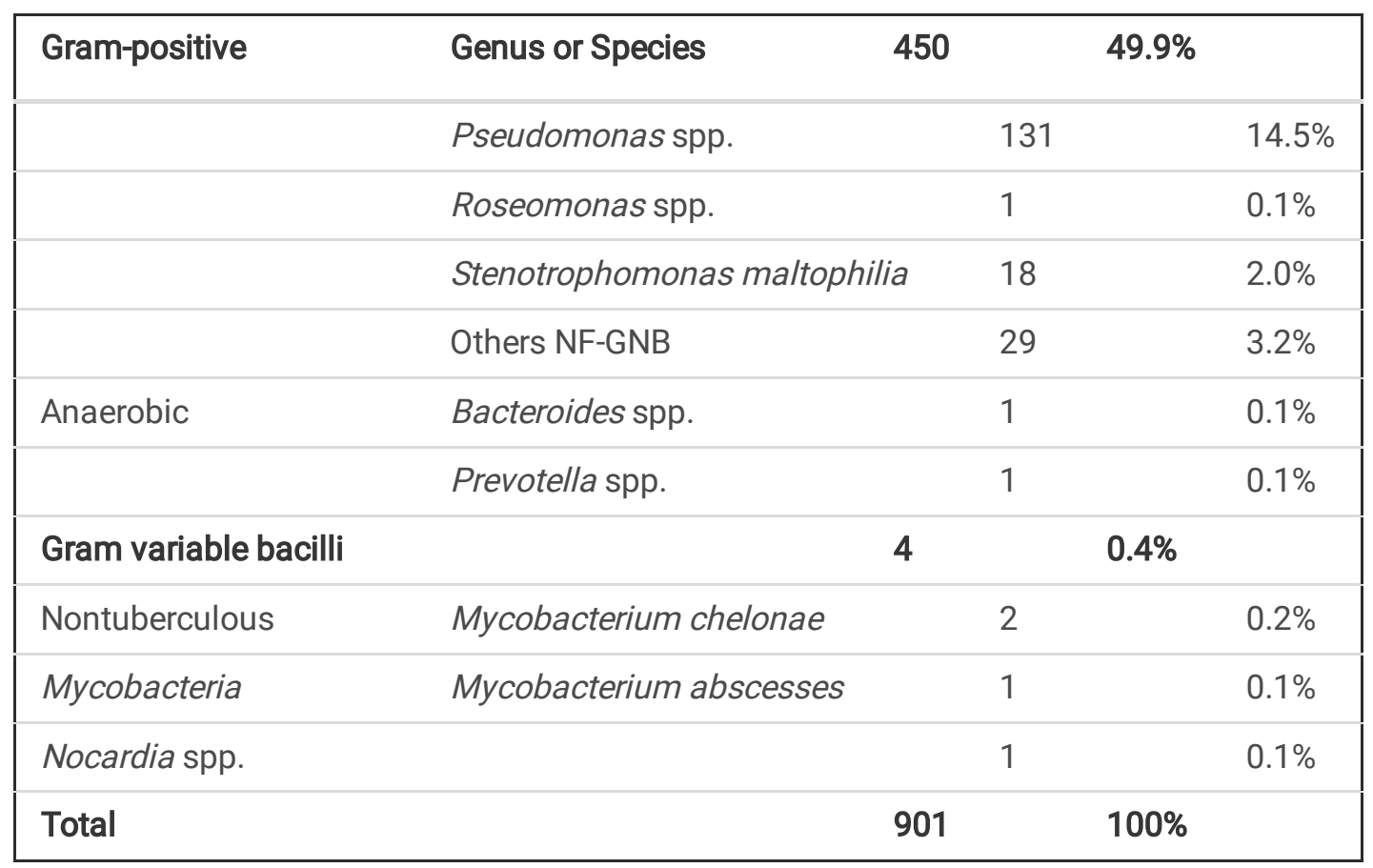

\section{Organismal spectrum}

Overall, $450(49.9 \%)$ of isolates were gram positive, $447(49.6 \%)$ were gram negative, and $4(0.4 \%)$ were gram variable. In the 450 gram-positive isolates, coagulase-negative staphylococci (CoNS) were the most commonly cultured bacterial organisms (158, 35.1\%), followed by streptococci $(100,22.2 \%)$, enterococci $(75,16.7 \%)$, and $S$. aureus $(70,15.6 \%)$. In the 447 gramnegative isolates, they were $K$. pneumoniae $(166,37.1 \%)$ and $P$. aeruginosa $(131,29.3 \%)$.

\section{Susceptibility of gram-positive isolates to vancomycin}

Table 2 presents the susceptibility of the gram-positive isolates to vancomycin. All gram-positive bacteria, with the exception of one Enterococcus faecium isolate, were susceptible to vancomycin, including CoNS, S. aureus, Enterococcus faecalis, and streptococci (Table 2). 
Table 2

Vancomycin susceptibility testing in Gram positive

bacteria. Note: CoNS = coagulase-negative Staphylococcus.

\begin{tabular}{|c|c|c|c|}
\hline & \multicolumn{3}{|c|}{ Vancomycin } \\
\hline \multicolumn{2}{|l|}{ Gram-positive bacteria } & Number & $\%$ \\
\hline \multicolumn{4}{|l|}{ Staphylococcus spp. } \\
\hline CoNS & 158 & $158 / 158$ & $100 \%$ \\
\hline S. aureus & 70 & $70 / 70$ & $100 \%$ \\
\hline Streptococcus spp. & 100 & $100 / 100$ & $100 \%$ \\
\hline Enterococcus spp. & 75 & $74 / 75$ & $98.7 \%$ \\
\hline Aerococcus spp. & 1 & $1 / 1$ & $100 \%$ \\
\hline Micrococcus spp. & 3 & $3 / 3$ & $100 \%$ \\
\hline Rothia mucilaginosa & 1 & $1 / 1$ & $100 \%$ \\
\hline Corynebacterium spp. & 9 & $9 / 9$ & $100 \%$ \\
\hline Cutibacterium acnes & 10 & $10 / 10$ & $100 \%$ \\
\hline Paenibacillus spp. & 1 & $1 / 1$ & $100 \%$ \\
\hline Bacillus spp. & 18 & $18 / 18$ & $100 \%$ \\
\hline \multirow[t]{2}{*}{ Clostridium spp. } & 4 & $4 / 4$ & $100 \%$ \\
\hline & 450 & $449 / 450$ & $99.8 \%$ \\
\hline
\end{tabular}

\section{Susceptibility of gram-negative isolates to amikacin and ceftazidime}

Table 3 presents the susceptibility of the gram-negative isolates to amikacin and ceftazidime. Overall, 96.9\% (413/426) were susceptible to ceftazidime and $93.7 \%$ (401/428) were susceptible to amikacin. Regarding the most commonly isolated gramnegative organisms, $98 \%$ of the K. pneumoniae isolates were susceptible to both ceftazidime and amikacin. Of the $P$. aeruginosa isolates, $98 \%$ and $95 \%$ were susceptible to ceftazidime and amikacin, respectively. Of the S. maltophilia isolates, $8 \%$ and $46 \%$ were susceptible to ceftazidime and amikacin, respectively. All the Haemophilus influenzae, Serratia marcescens, Enterobacter spp., Citrobacter spp., and E. coli isolates were susceptible to ceftazidime and amikacin. Among them, MDR gram-negative bacteria comprised K. pneumoniae (4/164, 2.4\%), A. baumannii (2/3,67\%), and $S$. maltophilia $(3 / 18,17 \%)$. The $S$. maltophilia isolates exhibited high resistance to ceftazidime $(11 / 12,94 \%)$ and amikacin $(7 / 13,54 \%)$. 
Table 3

Susceptibility of Gram-negative Bacterial Isolates to Ceftazidime and Amikacin. Note: NFGNB = Nonfermenting gramnegative bacilli; NT = not tested

\begin{tabular}{|c|c|c|c|c|c|}
\hline Gram-negative & Genus & Species & No. & Amikacin & Ceftazidime \\
\hline Cocci & Neisseria & & 4 & $1 / 1(100 \%)$ & $1 / 1(100 \%)$ \\
\hline Coccobacilli & Hemophilus & H. influenzae & 11 & $11 / 11(100 \%)$ & 11/11(100\%) \\
\hline \multicolumn{6}{|l|}{ Bacilli } \\
\hline \multicolumn{6}{|l|}{ Enterobacteriaceae } \\
\hline & Klebsiella & K. pneumoniae & 164 & $161 / 164(98 \%)$ & $160 / 164(98 \%)$ \\
\hline & & K. oxytoca & 2 & $2 / 2(100 \%)$ & $2 / 2(100 \%)$ \\
\hline & Citrobacter & C. freundii & 4 & $4 / 4(100 \%)$ & $4 / 4(100 \%)$ \\
\hline & & C. koseri & 4 & $4 / 4(100 \%)$ & $4 / 4(100 \%)$ \\
\hline & Enterobacter & E. aerogenes & 1 & $1 / 1(100 \%)$ & $1 / 1(100 \%)$ \\
\hline & & E. agglomerans & 2 & $2 / 2(100 \%)$ & $2 / 2(100 \%)$ \\
\hline & & E. cloacae & 11 & 11/11(100\%) & 11/11(100\%) \\
\hline & & E. gergoviae & 2 & $2 / 2(100 \%)$ & $2 / 2(100 \%)$ \\
\hline & Morganella & M. morganii & 2 & $2 / 2(100 \%)$ & $2 / 2(100 \%)$ \\
\hline & Proteus & P. mirabilis & 11 & 11/11(100\%) & 11/11(100\%) \\
\hline & & P. vulgaris & 1 & $1 / 1(100 \%)$ & $1 / 1(100 \%)$ \\
\hline & Salmonella & & 2 & NT & $2 / 2(100 \%)$ \\
\hline & Serratia & S. marcescens & 12 & 12/12(100\%) & 12/12(100\%) \\
\hline & Escherichia & E. coli & 13 & 13/13(100\%) & 13/13(100\%) \\
\hline Non-Enterobacteriaceae- & Aeromonas & A. hydrophilia & 3 & $3 / 3(100 \%)$ & $3 / 3(100 \%)$ \\
\hline fermentative & Vibrio & V. parahaemolyticus & 1 & NT & $1 / 1(100 \%)$ \\
\hline Non-Enterobacteriaceae- & Acinetobacter & A. baumannii & 3 & $1 / 3(33 \%)$ & $1 / 3(33 \%)$ \\
\hline \multirow[t]{11}{*}{ nonfermentative } & & A. Iwoffii & 1 & $1 / 1(100 \%)$ & $1 / 1(100 \%)$ \\
\hline & & A. pittii & 1 & $1 / 1(100 \%)$ & $1 / 1(100 \%)$ \\
\hline & Burkholderia & B. pseudomallei & 1 & $0 / 1(0 \%)$ & $1 / 1(100 \%)$ \\
\hline & & B. cepacia complex & 3 & $0 / 1(0 \%)$ & $3 / 3(100 \%)$ \\
\hline & Flavobacterium & & 3 & $3 / 3(100 \%)$ & $3 / 3(100 \%)$ \\
\hline & Moraxella & M. catarrhalis & 3 & NT & $3 / 3(100 \%)$ \\
\hline & & M. nonliquefaciens & 1 & NT & $1 / 1(100 \%)$ \\
\hline & Pseudomonas & P. aeruginosa & 123 & $117 / 123(95 \%)$ & $121 / 123(98 \%)$ \\
\hline & & P. fluorescens & 2 & $2 / 2(100 \%)$ & $2 / 2(100 \%)$ \\
\hline & & P. luteola & 1 & $1 / 1(100 \%)$ & $1 / 1(100 \%)$ \\
\hline & & P. stutzeri & 2 & $2 / 2(100 \%)$ & $2 / 2(100 \%)$ \\
\hline
\end{tabular}




\begin{tabular}{|llllll|}
\hline Gram-negative & Genus & Species & No. & Amikacin & Ceftazidime \\
\hline & & Non-identified & 3 & NT & NT \\
\hline Roseomonas & & 1 & $1 / 1(100 \%)$ & $0 / 1(0 \%)$ \\
& Stenotrophomonas & S. maltophilia & 18 & $6 / 13(46 \%)$ & $1 / 12(8 \%)$ \\
\hline Others NF-GNB & & 29 & $22 / 29(76 \%)$ & $25 / 29(86 \%)$ \\
\hline Anaerobic & Bacteroides & 1 & NT & NT \\
\hline & Prevotella & & 1 & NT & NT \\
\hline & & $\mathbf{4 4 7}$ & $\mathbf{4 0 1 / 4 2 8 ( 9 3 . 7 \% )}$ & $\mathbf{4 1 3 / 4 2 6 ( 9 6 . 9 \% )}$ \\
\hline
\end{tabular}

\section{Susceptibility of gram-variable isolates}

Two Mycobacterium chelonae, one Mycobacterium abscessus, and one Nocardia spp. isolate comprised the gram-variable isolates. Three Mycobacterium isolates were susceptible to amikacin but were not tested for susceptibility to ceftazidime. The Nocardia spp. isolate was not tested for susceptibility to amikacin and ceftazidime.

\section{MDR organisms}

Ten isolates $(10 / 901,1.1 \%)$, including one E. faecium, four K. pneumoniae, two A. baumannii, and three S. maltophilia, were MDR organisms. Table 4 shows the antibiotic susceptibility testing results for multidrug resistance. The $E$. faecium isolate was susceptible to linezolid and tigecycline but resistant to teicoplanin. ESBL production was detected in the four $K$. pneumoniae isolates, of which only one was susceptible to amikacin. All four isolates were susceptible to carbapenems (imipenem, meropenem, or ertapenem). One of the $A$. baumannii isolates was susceptible to imipenem. The other was resistant to meropenem but susceptible to colistin and tigecycline. All three MDR S. maltophilia isolates were susceptible to amikacin but resistant to ceftazidime, trimethoprim-sulfamethoxazole, and fluoroquinolones, including levofloxacin and moxifloxacin. 
Table 4

Antibiotic susceptibility testing in multidrug-resistant organisms. Note: CLA = clavulanic acid; I = intermediate; $\mathrm{S}$ = susceptible; SUL = sulbactam; $\mathrm{R}$ = resistant; TMP-SXT = trimethoprim-sulfamethoxazole; TZP= tazobactam.

\begin{tabular}{|c|c|c|c|c|c|c|c|c|c|c|}
\hline \multirow{3}{*}{$\begin{array}{l}\text { Organisms } \\
\text { Antibiotics } \\
\text { No. of isolates }\end{array}$} & \multirow{3}{*}{$\begin{array}{l}\text { Enterococcus } \\
\text { faecium } \\
1\end{array}$} & \multicolumn{3}{|c|}{ Klebsiella } & \multicolumn{3}{|c|}{ Acinetobacter } & \multicolumn{3}{|c|}{ Stenotrophomonas } \\
\hline & & \multicolumn{4}{|c|}{ pneumoniae } & \multicolumn{2}{|c|}{ baumannii } & \multicolumn{3}{|c|}{ maltophilia } \\
\hline & & 1 & 2 & 3 & 4 & 1 & 2 & 1 & 2 & 3 \\
\hline Penicillin & $\mathrm{R}$ & & & & & & & & & \\
\hline Ampicillin & $\mathrm{R}$ & $\mathrm{R}$ & $\mathrm{R}$ & $\mathrm{R}$ & & $\mathrm{R}$ & & & & \\
\hline Amoxicillin-CLA & & & & & $S$ & & & & & \\
\hline Ampicillin-SUL & & & & & & & $\mathrm{R}$ & & & \\
\hline Piperacillin & & $\mathrm{R}$ & $\mathrm{R}$ & S & $\mathrm{R}$ & $\mathrm{R}$ & $\mathrm{R}$ & & & \\
\hline Piperacillin-TZP & & & $S$ & & $S$ & & $\mathrm{R}$ & & & \\
\hline Vancomycin & $\mathrm{R}$ & & & & & & & & & \\
\hline Teicoplanin & $\mathrm{R}$ & & & & & & & & & \\
\hline Linezolid & S & & & & & & & & & \\
\hline Tigecycline & S & & & & & & $S$ & $\mathrm{R}$ & $\mathrm{R}$ & I \\
\hline Gentamicin & & $\mathrm{R}$ & $\mathrm{R}$ & $\mathrm{R}$ & $\mathrm{R}$ & $\mathrm{R}$ & $\mathrm{R}$ & & & \\
\hline High level GM & $\mathrm{R}$ & & & & & & & & & \\
\hline Amikacin & & $\mathrm{R}$ & $\mathrm{R}$ & $\mathrm{R}$ & $S$ & $\mathrm{R}$ & $\mathrm{R}$ & $S$ & $S$ & S \\
\hline Cefazolin & & $\mathrm{R}$ & $\mathrm{R}$ & $\mathrm{R}$ & $\mathrm{R}$ & $\mathrm{R}$ & & & & \\
\hline Cefuroxime & & $\mathrm{R}$ & $\mathrm{R}$ & $\mathrm{R}$ & $\mathrm{R}$ & $\mathrm{R}$ & & $\mathrm{R}$ & $\mathrm{R}$ & $\mathrm{R}$ \\
\hline Ceftriaxone & & $\mathrm{R}$ & $\mathrm{R}$ & $\mathrm{R}$ & $\mathrm{R}$ & $\mathrm{R}$ & $\mathrm{R}$ & & & \\
\hline Ceftazidime & & $\mathrm{R}$ & $\mathrm{R}$ & $\mathrm{R}$ & $\mathrm{R}$ & $\mathrm{R}$ & $\mathrm{R}$ & $\mathrm{R}$ & $\mathrm{R}$ & $\mathrm{R}$ \\
\hline Floxomef & & $\mathrm{R}$ & $\mathrm{R}$ & S & $S$ & $\mathrm{R}$ & & & & \\
\hline Cefepime & & & & & & & $\mathrm{R}$ & & & \\
\hline Aztreonam & & $\mathrm{R}$ & $\mathrm{R}$ & $\mathrm{R}$ & $\mathrm{R}$ & $\mathrm{R}$ & & & & \\
\hline TMP-SMX & & $\mathrm{R}$ & $\mathrm{R}$ & $\mathrm{R}$ & & $\mathrm{R}$ & & $\mathrm{R}$ & $\mathrm{R}$ & $\mathrm{R}$ \\
\hline Ciprofloxacin & & $S$ & $\mathrm{R}$ & $S$ & $S$ & $\mathrm{R}$ & $\mathrm{R}$ & & & \\
\hline Levofloxacin & & & & & & & & $\mathrm{R}$ & $\mathrm{R}$ & $\mathrm{R}$ \\
\hline Moxifloxacin & & & & & & & & $\mathrm{R}$ & $\mathrm{R}$ & $\mathrm{R}$ \\
\hline Imipenem & & $S$ & & S & & $S$ & & & & \\
\hline Meropenem & & & $\mathrm{S}$ & & & & $\mathrm{R}$ & & & \\
\hline Ertapenem & & & & & $S$ & & & & & \\
\hline Colistin & & & & & & & $S$ & & & \\
\hline
\end{tabular}




\section{Discussion}

Understanding of the various organisms causing bacterial endophthalmitis is critical to the development of effective treatments. Case studies on bacterial endophthalmitis have reported variability in the microbiological spectrum of causative pathogens according to the types of endophthalmitis and to the regions and countries where the studies were conducted. Bacterial endophthalmitis is caused by gram-positive and gram-negative organisms, as well as some gram-variable organisms. Empirically supported antibiotic treatments for infectious endophthalmitis are developed on the basis of likely causative organisms and their susceptibility patterns. In this study, the proportions of gram-positive and gram-negative bacteria (49.9\% and $49.6 \%$, respectively) were approximately equal, with gram-variable bacteria accounting for a considerably lower proportion $(0.4 \%)$. This is partially attributable to the large number of reported cases of endogenous $K$. pneumoniae endophthalmitis and $P$. aeruginosa keratitis-related endophthalmitis in Taiwan $4,6,10-12$. Our data differ from those of other published reviews of endophthalmitis isolates (Table 5) ${ }^{13-19}$. Gram-positive bacteria account for most bacterial isolates of endophthalmitis in studies conducted in the United States, Canada, and Austria, whereas gram-negative bacteria are predominant in India ${ }^{13-19}$. The higher proportion of gram-negative isolates observed in one study ${ }^{17}$ from India may be explained by trauma and environmental factors. In this study, 1.1\% (10/901) of the MDR bacterial isolates included one grampositive and nine gram-negative bacterial organisms.

Table 5

Review and comparison of bacterial endophthalmitis isolates. Note: $\mathrm{BPEI}=\mathrm{Bascom}$ Palmer Eye Institute; $\mathrm{CGMH}=\mathrm{Chang}$ Gung Memorial Hospital; N/A = not available; NYEEI = New York Eye and Ear Infirmary; UPMC = University of Pittsburgh Medical Center.

\begin{tabular}{|c|c|c|c|c|c|c|c|c|}
\hline & UPMC $^{19}$ & NYEEI $^{13}$ & BPEI $^{15}$ & Toronto $^{18}$ & Hyderabad ${ }^{17}$ & Hyderabad $^{14}$ & Queensland ${ }^{16}$ & $\mathrm{CGMH}^{*}$ \\
\hline & USA & USA & USA & Canada & India & India & Australia & Taiwan \\
\hline Study period & $\begin{array}{l}1993- \\
2015\end{array}$ & $\begin{array}{l}1987- \\
2011\end{array}$ & $\begin{array}{l}2002- \\
2011\end{array}$ & $\begin{array}{l}2000- \\
2009\end{array}$ & 1991-2015 & $2010-2013$ & 1998-2013 & $\begin{array}{l}1996- \\
2019\end{array}$ \\
\hline $\begin{array}{l}\text { No. of } \\
\text { isolates }\end{array}$ & 665 & 943 & 375 & 265 & 2840 & 196 & 193 & 901 \\
\hline $\begin{array}{l}\text { Gram- } \\
\text { positive } \\
\text { isolates }\end{array}$ & $92.9 \%$ & $89.2 \%$ & $87.2 \%$ & $90.2 \%$ & $68.7 \%$ & $37.2 \%$ & $84.5 \%$ & $49.9 \%$ \\
\hline $\begin{array}{l}\text { Susceptibility } \\
\text { to } \\
\text { vancomycin }\end{array}$ & $99.7 \%$ & $99.7 \%$ & $100.0 \%$ & $99.6 \%$ & $96 \%$ & $100.0 \%$ & $100.0 \%$ & $99.8 \%$ \\
\hline $\begin{array}{l}\text { Gram- } \\
\text { negative } \\
\text { isolates }\end{array}$ & $7.1 \%$ & $10.8 \%$ & $12.8 \%$ & $9.8 \%$ & $32.3 \%$ & $62.8 \%$ & $15.5 \%$ & $49.6 \%$ \\
\hline $\begin{array}{l}\text { Susceptibility } \\
\text { to amikacin }\end{array}$ & $95.7 \%$ & $92.9 \%$ & $\mathrm{~N} / \mathrm{A}$ & $\mathrm{N} / \mathrm{A}$ & $64-67 \% \dagger$ & $87.0 \%$ & $100.0 \%$ & $93.7 \%$ \\
\hline $\begin{array}{l}\text { Susceptibility } \\
\text { to } \\
\text { ceftazidime }\end{array}$ & $93.6 \%$ & $91.5 \%$ & $100.0 \%$ & $100.0 \%$ & $69-38 \% \dagger$ & $82.0 \%$ & $100.0 \%$ & $96.9 \%$ \\
\hline
\end{tabular}

*Including 4 gram-variable isolates

$+2005 \rightarrow 2015$

As mentioned, the most commonly cultured gram-positive isolates were CoNS, followed by Streptococcus spp., Enterococcus spp., and $S$. aureus. These results are consistent with those from previous studies ${ }^{13-17}$. However, a higher proportion of Enterococcus spp., especially E. faecalis, was noted in this study, and it resulted from more $E$. faecalis endophthalmitis cases in Taiwan 20,21. Gram-positive vancomycin-resistant endophthalmitis has become a critical clinical issue worldwide; this is 
indicated by the fact that Enterococcus spp. and Staphylococcus spp. were the most commonly reported organisms ${ }^{7}$. The rate of gram-positive vancomycin resistance in the present study was $0.2 \%$. All Staphylococcus spp. isolates were susceptible to vancomycin, but 1 of the 75 (1.3\%) Enterococcus isolates, E. faecium, was resistant to vancomycin. Several case reports have examined vancomycin-resistant enterococcal endophthalmitis ${ }^{22-24}$. E. faecalis was less commonly resistant to vancomycin. In other case reports of endophthalmitis, E. casseliflavus ${ }^{25}$ and E. faecium ${ }^{2}$ exhibited higher rates of resistance to vancomycin. In a literature review ${ }^{7}$ of 27 types of vancomycin-resistant gram-positive bacteria, Enterococcus spp., CoNS, $S$. aureus, and Bacillus spp. were the most common organisms.

In this study, $3.1 \%$ and $6.3 \%$ of the gram-negative bacteria was resistant to ceftazidime and amikacin, respectively. In these resistant isolates, most gram-negative bacteria were resistant to either ceftazidime or amikacin. However, five isolates $(5 / 447$, $1.1 \%)$ were resistant to both ceftazidime and amikacin.

Apart from four K. pneumoniae isolates, most bacteria in the Enterobacteriaces family were susceptible to amikacin and ceftazidime. By comparison, nonfermenting gram-negative bacteria not belonging to the Enterobacteriaces family were more likely to be resistant to ceftazidime and amikacin. P. aeruginosa had a comparable degree of susceptibility to ceftazidime $(98 \%)$ and amikacin (95\%). By contrast, the susceptibility of S. maltophilia to ceftazidime and amikacin differed substantially ( $8 \%$ and $46 \%$, respectively).

Among the MDR bacteria, E. faecium was susceptible to linezolid and tigecycline. The nine MDR gram-negative bacterial isolates comprised K. pneumoniae (4/164, 2.4\%), A. baumannii (2/3,67\%), and S. maltophilia (3/18, 17\%). The ceftazidime resistance of $K$. pneumoniae, a member of the Enterobacteriaceae family, was indicated by the presence of ESBL production. The ESBL-producing gram-negative bacteria were resistant to all third-generation cephalosporins. Four MDR K. pneumoniae isolates were susceptible to carbapenems (imipenem, meropenem, or ertapenem). One of the A. baumannii isolates was susceptible to carbapenem, and the other was susceptible to colistin and tigecycline. All three MDR S. maltophilia isolates were susceptible to amikacin but resistant to ceftazidime, trimethoprim-sulfamethoxazole, and fluoroquinolones, including levofloxacin and moxifloxacin. In a case report of carbapenemase-producing K. pneumoniae endophthalmitis by Zhou et al. ${ }^{8}$, the $K$. pneumoniae isolate was resistant to amikacin, ceftazidime, and carbapenems but susceptible to polymyxin $E$ and tigecycline. Roy et al. ${ }^{26}$ noted that four (100\%) A. baumannii isolates were resistant to ceftazidime and one (25\%) was resistant to amikacin. All four of those $A$. baumannii isolates were susceptible to ciprofloxacin ${ }^{26}$. Although $S$. maltophilia was typically susceptible to trimethoprim-sulfamethoxazole, levofloxacin, and moxifloxacin, Ji et al. ${ }^{5}$ reported on 14 patients with MDR S. maltophilia postcataract endophthalmitis. All eight $S$. maltophilia isolates were resistant to amikacin, imipenem, and ciprofloxacin but not to levofloxacin ${ }^{5}$. Five isolates were resistant to ceftazidime. ${ }^{5}$

Most bacteria are typically susceptible to vancomycin, ceftazidime, and amikacin at a concentration of $\leq 10 \mu \mathrm{g} / \mathrm{mL}$. Interpretive standards for dilution susceptibility testing from the US Clinical and Laboratory Standards Institute indicate that the minimal inhibitory concentrations of vancomycin, ceftazidime, and amikacin are $\leq 4, \leq 8$, and $\leq 6 \mu \mathrm{g} / \mathrm{mL}$, respectively 27 . The resistance concentrations of vancomycin, ceftazidime, and amikacin are $\geq 32, \geq 32$, and $\geq 64 \mu \mathrm{g} / \mathrm{mL}$, respectively 27 . Intravitreal antibiotic injection is the principal treatment for infectious endophthalmitis. In vitro resistance patterns may not be identical with in vivo susceptibility, and routinely administered intravitreal antibiotics typically deliver intraocular antibiotic concentrations that are considerably higher than the minimal inhibitory concentrations of most organisms. When patients' conditions do not stabilize or improve after initial intravitreal antibiotic injection, additional injections of alternative antibiotics should be considered according to results in vitro susceptibility testing.

This study has some limitations. First, this was a single-center retrospective case series. Second, some gram-negative isolates were not routinely tested at our hospital for susceptibility to amikacin and ceftazidime. Third, we did not retrieve accurate, detailed information on various types of endophthalmitis and visual outcomes, which may interest readers. Fourth, we did not analyze trends in the patterns of antibiotic susceptibility given the relatively small number of annual cases. Finally, we did not further analyze the MDR bacteria for specific resistance genes. Nevertheless, our findings contribute to the 
literature in providing a thorough analysis of the antimicrobial susceptibility of bacterial isolates from all patients diagnosed as having bacterial endophthalmitis over 23 years at a tertiary medical center.

In conclusion, the most commonly detected organisms in the gram-positive and gram-negative bacteria were CoNS and $K$. pneumoniae, respectively. MDR bacterial endophthalmitis was not common. Vancomycin remains the antibiotic of choice for the treatment of gram-positive endophthalmitis. Amikacin and ceftazidime appear to provide approximately equal gramnegative coverage.

\section{Declarations}

\section{Acknowledgments}

The authors wish to acknowledge the support of the Taiwan Ministry of Science and Technology MOST 106-2314-B-182A041, and Chang Gung Memorial Hospital CMRPG3F1243.

\section{Author Contributions}

K.-J.C. designed the study, performed statistical analysis, wrote the manuscript, and approved the final manuscript for publication; M.-H. S. collected and analyzed the data; H.-C.H., H.-C. C., Y.-P. C., N.-K. W., L. L., W.-C. W., H.-D. C.,E.-Y.K. and C.-C. L. all provided patients' data and assisted in data collection. All authors have reviewed the manuscript.

\section{Competing Interests}

The authors declare no competing interests.

\section{References}

1. Boucher, H. W. et al. Bad bugs, no drugs: no ESKAPE! An update from the Infectious Diseases Society of America. Clinical infectious diseases : an official publication of the Infectious Diseases Society of America 48, 1-12, doi:10.1086/595011 (2009).

2. Bains, H. S., Weinberg, D. V., Feder, R. S. \& Noskin, G. A. Postoperative vancomycin-resistant Enterococcus faecium endophthalmitis. Archives of ophthalmology (Chicago, III. : 1960) 125, 1292-1293, doi:10.1001/archopht.125.9.1292 (2007).

3. Chen, K. J. et al. Endophthalmitis caused by Acinetobacter baumannii: report of two cases. Journal of clinical microbiology 46, 1148-1150, doi:10.1128/jcm.01604-07 (2008).

4. Chen, K. J. et al. Endogenous Klebsiella endophthalmitis associated with Klebsiella pneumoniae pneumonia. Ocular immunology and inflammation 17, 153-159, doi:10.1080/09273940902752250 (2009).

5. Ji, Y. et al. Post-cataract endophthalmitis caused by multidrug-resistant Stenotrophomonas maltophilia: clinical features and risk factors. BMC ophthalmology 15, 14, doi:10.1186/1471-2415-15-14 (2015).

6. Li, Y. H. et al. Infectious Sources, Prognostic Factors, and Visual Outcomes of Endogenous Klebsiella pneumoniae Endophthalmitis. Ophthalmology. Retina 2, 771-778, doi:10.1016/j.oret.2017.11.013 (2018).

7. Relhan, N. et al. Endophthalmitis caused by Gram-positive organisms with reduced vancomycin susceptibility: literature review and options for treatment. The British journal of ophthalmology 100, 446-452, doi:10.1136/bjophthalmol-2015307722 (2016).

8. Zhou, Y., Wang, X., Shen, J., Lu, Z. \& Liu, Y. Endogenous Endophthalmitis Caused by Carbapenem-Resistant Hypervirulent Klebsiella Pneumoniae: A Case Report and Literature Review. Ocular immunology and inflammation 27, 1099-1104, doi:10.1080/09273948.2018.1502786 (2019).

9. Clinical and Laboratory Standards Institute. Performance standards for antimicrobial disk susceptibility tests; approved standard-12th ed. M02-A13. Clinical and Laboratory Standards Institute, Wayne, Pa. 
10. Chen, Y. H. et al. Prognostic Factors and Visual Outcomes of Pyogenic Liver Abscess-Related Endogenous Klebsiella pneumoniae Endophthalmitis: A 20-year retrospective review. Scientific reports 9, 1071, doi:10.1038/s41598-018-37643-y (2019).

11. Chen, K. J. et al. Endophthalmitis caused by Pseudomonas aeruginosa in Taiwan. Retina (Philadelphia, Pa.) 31, 11931198, doi:10.1097/IAE.0b013e3181fbce5c (2011).

12. Chen, K. J., Wu, W. C., Sun, M. H., Lai, C. C. \& Chen, T. L. Pseudomonas endophthalmitis. Ophthalmology 117, 1657-1658; author reply 1658-1659, doi:10.1016/j.ophtha.2010.04.011 (2010).

13. Gentile, R. C. et al. Microbiological spectrum and antibiotic sensitivity in endophthalmitis: a 25-year review. Ophthalmology 121, 1634-1642, doi:10.1016/j.ophtha.2014.02.001 (2014).

14. Reddy, A. K. et al. Susceptibility of bacterial isolates to vancomycin and ceftazidime from patients with endophthalmitis: Is there a need to change the empirical therapy in suspected bacterial endophthalmitis? International ophthalmology 35, 37-42, doi:10.1007/s10792-014-0015-9 (2015).

15. Schimel, A. M., Miller, D. \& Flynn, H. W., Jr. Endophthalmitis isolates and antibiotic susceptibilities: a 10-year review of culture-proven cases. American journal of ophthalmology 156, 50-52.e51, doi:10.1016/j.ajo.2013.01.027 (2013).

16. Moloney, T. P. \& Park, J. Microbiological isolates and antibiotic sensitivities in culture-proven endophthalmitis: a 15-year review. The British journal of ophthalmology 98, 1492-1497, doi:10.1136/bjophthalmol-2014-305030 (2014).

17. Joseph, J. et al. Trends in microbiological spectrum of endophthalmitis at a single tertiary care ophthalmic hospital in India: a review of 25 years. Eye (London, England) 33, 1090-1095, doi:10.1038/s41433-019-0380-8 (2019).

18. Assaad, D. et al. Bacterial endophthalmitis: 10-year review of the culture and sensitivity patterns of bacterial isolates. Canadian journal of ophthalmology. Journal canadien d'ophtalmologie 50, 433-437, doi:10.1016/j.jcjo.2015.07.013 (2015).

19. Kodati, S., Eller, A. W. \& Kowalski, R. P. The Susceptibility of Bacterial Endophthalmitis Isolates to Vancomycin, Ceftazidime, and Amikacin: a 23 Year-Review. Ophthalmology. Retina 1, 206-209, doi:10.1016/j.oret.2016.11.010 (2017).

20. Chen, K. J. et al. Postcataract endophthalmitis caused by Enterococcus faecalis. Ocular immunology and inflammation 17, 364-369, doi:10.3109/09273940903105110 (2009).

21. Chen, K. J. et al. Enterococcus faecalis Endophthalmitis: Clinical Settings, Antibiotic Susceptibility, and Management Outcomes. Microorganisms 9, doi:10.3390/microorganisms9050918 (2021).

22. Dave, V. P. et al. ENTEROCOCCUS ENDOPHTHALMITIS: Clinical Settings, Antimicrobial Susceptibility, and Management Outcomes. Retina (Philadelphia, Pa.) 40, 898-902, doi:10.1097/iae.0000000000002462 (2020).

23. Rishi, E., Rishi, P., Nandi, K., Shroff, D. \& Therese, K. L. Endophthalmitis caused by Enterococcus faecalis: a case series. Retina (Philadelphia, Pa.) 29, 214-217, doi:10.1097/IAE.0b013e31818eccc7 (2009).

24. Tang, C. W., Cheng, C. K. \& Lee, T. S. Community-acquired bleb-related endophthalmitis caused by vancomycin-resistant enterococci. Canadian journal of ophthalmology. Journal canadien d'ophtalmologie 42, 477-478 (2007).

25. Low, J. R., Teoh, C. S., Chien, J. M. \& Huang, E. H. Enterococcus casseliflavus endophthalmitis due to metallic intraocular foreign body. Eye (London, England) 29, 840-841, doi:10.1038/eye.2014.332 (2015).

26. Roy, R. et al. Endophthalmitis caused by Acinetobacter baumanni: a case series. Eye (London, England) 27, 450-452, doi:10.1038/eye.2012.277 (2013).

27. Performance Standards for Antimicrobial Susceptibility Testing. Clinical and Laboratory Standards Institute (CLSI). M100 28th edition. January 2018. 